\section{Wechselwirkungseffekt, quantitativer}

R.-D. Hilgers ${ }^{1}$, N. Heussen ${ }^{1}$ und S. Stanzel ${ }^{2}$

${ }^{1}$ Institut für Medizinische Statistik, Universitätsklinikum der RWTH Aachen, Aachen, Deutschland

${ }^{2}$ DKFZ Heidelberg, Heidelberg, Deutschland

Synonym(e) Quantitative Wechselwirkung

Englischer Begriff quantitative interaction; quantitative interaction effect

Definition Man spricht von einem quantitativen Wechselwirkungseffekt, wenn die Größenordnung, aber nicht die Richtung des Behandlungseffekts zwischen den Gruppen variiert.

Beschreibung Man betrachte einen Versuch, bei dem aus 2 verschiedenen Laboratorien (I, II) Messergebnisse (s. \ Mess- ergebnis) zu einem Laborparameter vorliegen, die mit jeweils 2 unterschiedlichen Messmethoden (s. - Messmethode) (A, B) ermittelt wurden. Eine quantitative Wechselwirkung bzw. ein quantitativer Wechselwirkungseffekt liegt genau dann vor, wenn die Differenz der mittleren Messwerte unter der Methode A gegenüber der Methode B sich für Labor I lediglich im Ausmaß, jedoch nicht in der Richtung (Vorzeichen) von der entsprechenden Differenz für Labor II unterscheidet.

Oft ist das Ausmaß des Unterschieds zwischen den Differenzen, also die quantitative Wechselwirkung, abhängig vom Maßstab der Messung.

\section{Literatur}

Day S (1999) Dictionary for clincal trials. Wiley, New York

Fisher LD, van Belle G (1993) Biostatistics - a methodology for the health sciences. Wiley, New York 